\title{
Myrtol improves post-traumatic knee osteoarthritis by regulation of reactive oxygen species, transforming growth factor $\beta 1$ and apoptosis in a mouse model
}

\author{
LIQUN DUAN, WENZHI ZHANG, FENG ZHANG and HAIPING CAI
}

\author{
Department of Orthopedics, Anhui Provincial Hospital, Hefei, Anhui 230001, P.R. China
}

Received February 7, 2016; Accepted February 10, 2017

DOI: $10.3892 / \mathrm{etm} .2017 .5367$

\begin{abstract}
The present study tested whether myrtol improves post-traumatic knee osteoarthritis (PTKO) by regulating the reactive oxygen species (ROS), transforming growth factor $\beta 1$ (TGF- $\beta 1$ ) and apoptosis in a mouse model. PTKO model mice were administered with 150,300 or $450 \mathrm{mg} / \mathrm{kg}$ myrtol for 8 weeks. ELISA analysis was used to measure tumor necrosis factor- $\alpha$, interleukin-6, malondialdehyde, superoxide dismutase, reactive oxygen species and TGF- $\beta 1$ levels. Caspase-3 and Bax protein expressions were analyzed using western blot analysis. In the current study, treatment with myrtol improved the tissue damage and osteoarthritis score, while it also reversed the subchondral bone thickness, subchondral bone density, trabecular bone volume/relative trabecular bone volume ratio and trabecular bone spacing in PTKO mice. The activity of tumor necrosis factor $\alpha$, interleukin-6, TGF- $\beta 1$, malondialdehyde, superoxide dismutase and ROS were effectively inhibited, and the protein expression of caspase-3 and Bax were clearly suppressed by treatment with myrtol in a mouse model of PTKO. In conclusion, the results demonstrated that myrtol treatment improved PTKO through the suppression of inflammation, oxidative stress, ROS, TGF- $\beta 1$ and Bax/caspase- 3 in mice, and myrtol may be a potential agent for clinical therapy.
\end{abstract}

\section{Introduction}

Post-traumatic knee osteoarthritis (PTKO) is a common disease presenting joint degenerative changes (1). PTKO is most frequently detected on the elderly population, with an estimated 15 million cases in China (2). Survey data in the United States also demonstrated that PTKO is the second most common cause of disability in males $>50$ years old, after cardiovascular

Correspondence to: Dr Liqun Duan, Department of Orthopedics, Anhui Provincial Hospital, 17 Lujiang Road, Hefei, Anhui 230001, P.R. China

E-mail: liqunduanmu@163.com

Key words: myrtol, post-traumatic knee osteoarthritis, reactive oxygen species, transforming growth factor- $\beta 1$ disease (3). Thus, prevention and treatment of PTKO have become one of the most difficult issues in clinical practice at present (3).

Transforming growth factor $\beta 1$ (TGF- $\beta 1$ ) is a member of the TGF- $\beta$ superfamily and a type of multifunctional polypeptide growth factor (4). It participates in the proliferation and differentiation processes of diversified cell types, such as chondrocytes, epithelial cells and fibroblasts, and has essential regulating effects in the developing process of embryo, organs and cartilage. Studies have demonstrated that, in the chondrocytes of rabbits and cattle, TGF- $\beta 1$ can regulate the synthesis of proteoglycan $(4,5)$. In addition, TGF- $\beta 1$ has the ability to promote synthesis of type II collagen by reducing consumption of proteoglycan in order to repair the cartilage (6). Therefore, it can be seen that TGF- $\beta 1$ serves an important regulatory role in the process of maintaining chondrocytes and cartilage (7).

PTKO is a chronic autoimmune disease characterized by joint synovitis, and its early lesion mainly presents on the synovial membrane (8). Synovial cells are subject to rapid proliferation due to autoimmune response resulting from an external stimulus, such as infection or trauma (9); thus, the infiltration of diversified inflammatory cells on the synovial membrane is enhanced (9). In addition, a large number of cytokines, inflammatory proteins and various antibodies jointly mediate the inflammation-induced tissue damage in PTKO (10). During the pathological process of PTKO, interleukin (IL)-1 $\beta$, tumor necrosis factor (TNF)- $\alpha$, IL- 6 and other proinflammatory cytokines, as well as the inflammatory mediator prostaglandin E2 (PGE2), serve an important role (11).

Pharmacological and clinical studies in China and abroad have revealed that myrtol standardized, a natural phytomedicine used for the therapy of acute or chronic sinusitis and bronchitis, has high safety, reduced adverse reactions and good clinical efficacy (12). Furthermore, it has extensive clinical efficacy on respiratory system diseases. Domestic clinical studies have shown that its clinical efficacy is similar to the total curative effect of the import gel myrtol forte group (12). Furthermore, pharmacological and pharmacodynamics studies have demonstrated that myrtol standardized has expectorant anti-inflammatory effects, relieving cough and asthma, and reported reduced toxicity and side-effects (13).

The present study investigated whether myrtol improves PTKO through regulation of reactive oxygen species (ROS), TGF- $\beta 1$ and apoptosis in mice. 


\section{Materials and methods}

Animals. A total of $60 \mathrm{C} 57 \mathrm{BL} / 6 \mathrm{~N}$ female mice $(20 \pm 1 \mathrm{~g}$; 8-weeks-old) were obtained from Laboratory Animal Center of Anhui Medical University (Hefei, China) and divided into five cages. All mice were kept under controlled environmental conditions (temperature, $23 \pm 1^{\circ} \mathrm{C}$; $50-60 \%$ relative humidity; $12 \mathrm{~h}$ alternate light-dark cycles). All experiments have been approved by the Ethics Committee of Anhui Provincial Hospital (Hefei, China).

PTKO model and myrtol administration. All mice were randomly divided into five groups, including the control (8 mice), PTKO (16 mice), low dose myrtol-treated (12 mice), medium dose myrtol-treated (12 mice) and high dose myrtol-treated (12 mice) groups. This loading protocol causes alendronate rupture with associated avulsion fracture from the distal femur (14). Mice were anesthetized with intraperitoneal injection of 3\% sodium pentobarbital (Sigma-Aldrich, St. Louis, MO, USA). Knee injury was induced by an electromagnetic materials testing system (ELF 3200; Bose, Eden Prairie, MN, USA), with $1 \mathrm{~mm} / \mathrm{sec}$ loading rate of a single dynamic overload cycle to a target compressive load of $12 \mathrm{~N}$. Knee injury in mice was performed by the release of compressive force during loading and by an audible click. Mice in the low, medium and high myrtol dose groups were administered with 150,300 or $450 \mathrm{mg} / \mathrm{kg}$ myrtol (G.Pohl-Boskamp $\mathrm{GmbH}$, Hohenlockstedt, Germany), respectively, every day for 8 weeks following knee injury. In the control group, rats were anesthetized only. The control and PTKO groups were given equal volume of saline.

Hematoxylin and eosin staining. At 8 weeks following myrtol treatment, rats were sacrificed, and knee tissue samples were acquired and fixed in $10 \%$ paraformaldehyde for $24 \mathrm{~h}$ at room temperature. Tissues were paraffin-embedded and stored at $4^{\circ} \mathrm{C}$ until required. The tissue samples from each group were then cut into $4 \mu \mathrm{m}$ sections using a paraffin slicing machine (RM2235; Leica, Wetzlar, Germany), stained with hematoxylin and eosin, and observed under light microscopy (BH3-MJL; Olympus Corp., Tokyo, Japan). Osteoarthritis score was determined using the modified Mankin scoring system (15). Next, the synovial extension score was determined as absence (score 0 ) and presence (score 1) of synovial tissue outgrowth parallel to the surface of the meniscus (score 2), the meniscus adjacent to the femoral and tibial surfaces in both the anterior (score 3) and posterior regions (score 4).

Micro-computed tomography (micro-CT) analyses. Knee tissue samples were acquired, placed into $10 \%$ paraformaldehyde for $24 \mathrm{~h}$ and scanned using a micro-CT system (vivaCT; Scanco Medical, Basserdorf, Switzerland). The subchondral bone thickness $(\mathrm{mm})$, subchondral bone density (mg hydroxyapatite $\left./ \mathrm{cm}^{3}\right)$, trabecular bone volume $\left(\mathrm{cm}^{3}\right)$, relative trabecular bone volume (BV/TV) ratio and trabecular bone spacing $(\mathrm{mm})$ were analyzed.

ELISA analysis of TNF- $\alpha, I L-6$, malondialdehyde (MDA), superoxide dismutase (SOD), ROS and TGF- $\beta 1$ levels. A total of $200 \mu \mathrm{l}$ of blood was collected from mice in each group immediately following sacrifice and separated using centrifugation at 5,000 $\mathrm{xg}$ for $10 \mathrm{~min}$ at $4^{\circ} \mathrm{C}$. Blood serum was acquired and analyzed for TNF- $\alpha$ (cat. no. E-CL-M0047c), IL-6 (cat. no. E-CL-M0042c), MDA (cat. no. E-EL-0060c), SOD (cat. no. E-EL-M2424c) and TGF- $\beta 1$ (cat. no. E-EL-M0051c) levels using ELISA kits (Wuhan Huamai Biotech Co., Wuhan, China), according to the manufacturer's instructions. ROS was measured using Reactive oxygen species Assay kit (cat. no. E004; Nanjing Jiancheng Biology Engineering Institute, Nanjing, China). Absorbance was measured using an M200 plate reader (Techan Trading AG, Mannedorf, Switzerland) at $450 \mathrm{~nm}$.

Western blot analysis. Knee tissue samples were acquired and prepared in a lysis buffer (Beyotime Institute of Biotechnology, Haimen, China). Following homogenization, the samples were centrifuged at 10,000 $\mathrm{x}$ g for $30 \mathrm{~min}$ and the protein contents were estimated with a BCA protein assay kit (Beyotime Institute of Biotechnology). Protein extraction was resolved by $12 \%$ sodium dodecyl sulphate-polyacrylamide gel electrophoresis, and samples were then transferred onto a nitrocellulose membrane (Sigma-Aldrich). The nitrocellulose membrane was blocked with $5 \%$ bovine serum albumin or $5 \%$ non-fat dry milk. the nitrocellulose membrane was incubated with anti-caspase-3 (sc-7148; 1:4,000), anti-Bax (sc-6236; 1:3,000) and $\beta$-actin (sc-130656; 1:5,000; all from Santa Cruz Biotechnology, Dallas, TX, USA) antibodies at $4^{\circ} \mathrm{C}$ for $12 \mathrm{~h}$. Membranes were subsequently washed with PBS for three times at $5 \mathrm{~min}$ and incubated with mouse anti-rabbit IgG-HRP (sc-2357; 1:5,000, Santa Cruz Biotechnology) for $1 \mathrm{~h}$ at $37^{\circ} \mathrm{C}$ and visualized by an enhanced chemiluminescence assay kit (KGP1125; Nanjing KeyGen Biotech. Co., Ltd., Nanjing, China). The protein expression was scanned with a densitometer and analyzed with Multi-Analyst software 3.0 (Bio-Rad Laboratories, Inc, Hercules, CA, USA).

Statistical analysis. Results are expressed as the mean \pm standard deviation. Statistically significant differences were evaluated using one-way analysis of variance with SPSS version 13.0 software (SPSS, Inc., Chicago, IL, USA), and were indicated by $\mathrm{P}<0.05$.

\section{Results}

Myrtol reduces osteoarthritis in the PTKO mouse model. After treatment for 8 weeks, the extent of knee osteoarthritis in the PTKO model group was higher compared with that of the control group, as observed by hematoxylin and eosin staining (Fig. 1). Low dose myrtol treatment did not have a significant effect on knee osteoarthritis in PTKO mice (Fig. 1). However, treatment with medium or high dose of myrtol significantly reduced the knee osteoarthritis in PTKO mice (Fig. 1; $\mathrm{P}=0.0034$ and $\mathrm{P}=0.0012$, respectively).

Myrtol improves knee tissue score in PTKO model. The osteoarthritis and synovial extension scores of each knee tissue sample were graded in order to examine the effect of myrtol treatment on PTKO. The osteoarthritis score of the PTKO model group was significantly higher compared with that of the control group $(\mathrm{P}=0.0016)$, and low dose myrtol treatment had no significant effect on this score in PTKO mice (Fig. 2A). 

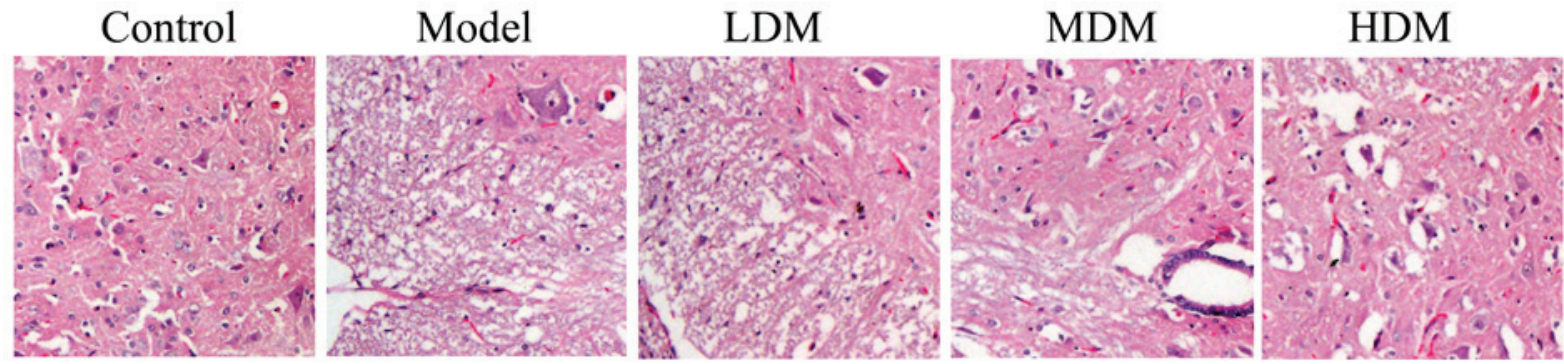

Figure 1. Myrtol improves knee tissue samples in a PTKO mouse model. PTKO, post-traumatic knee osteoarthritis; LDM, low dose myrtol; MDM, medium dose myrtol; HDM, high dose myrtol.
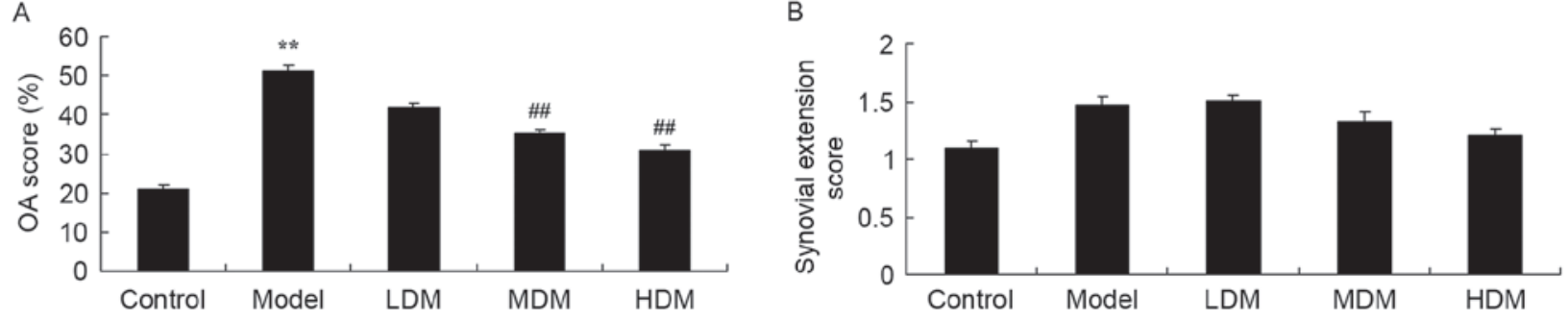

Figure 2. Myrtol improves knee tissue score in a PTKO mouse model. Effect on (A) OA score and (B) synovial extension score from a maximum of $4 .{ }^{* *} \mathrm{P}<0.01$ vs. control model group; ${ }^{\# /} \mathrm{P}<0.01$ vs. PTKO group. PTKO, post-traumatic knee osteoarthritis; LDM, low dose myrtol; MDM, medium dose myrtol; HDM, high dose myrtol; OA, osteoarthritis.
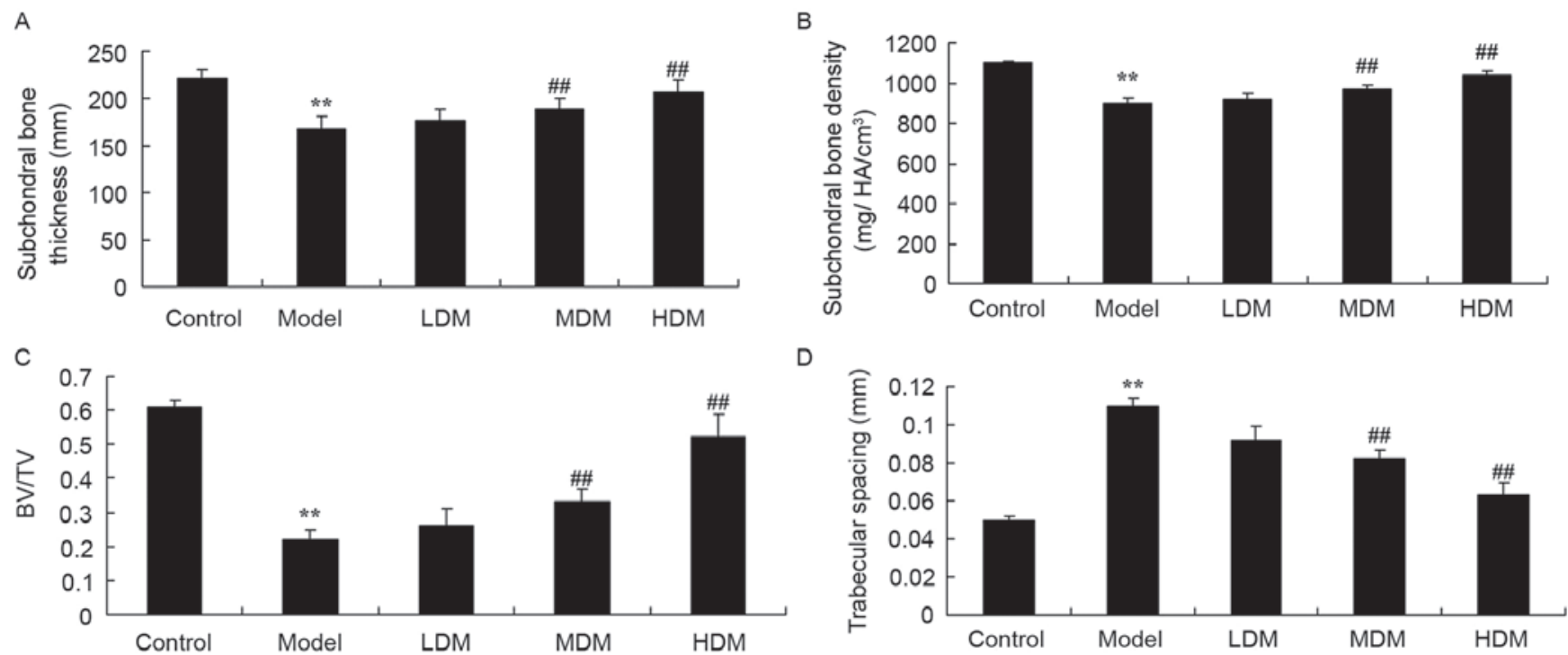

Figure 3. Myrtol improves the results of micro-computed tomography analyses in a PTKO mouse model. The (A) subchondral bone thickness, (B) subchondral bone density, (C) BV/TV and (D) trabecular bone spacing in mice are shown. ${ }^{* *} \mathrm{P}<0.01$ vs. control model group; ${ }^{\# /} \mathrm{P}<0.01$ vs. PTKO group. PTKO, post-traumatic knee osteoarthritis; LDM, low dose myrtol; MDM, medium dose myrtol; HDM, high dose myrtol; BV/TV, trabecular bone volume/relative trabecular bone volume.

However, treatment with medium or high dose of myrtol significantly inhibited the osteoarthritis score in PTKO mice (Fig. 2A; $\mathrm{P}=0.0066$ and $\mathrm{P}=0.0049$, respectively). By contrast, the synovial extension score was not significantly different in the PTKO and myrtol-treated groups in comparison with the control (Fig. 2B).

Myrtol improves the results of micro-CT analyses in the PTKO model. The subchondral bone thickness (Fig. 3A; $\mathrm{P}=0.0059$ ), subchondral bone density (Fig. $3 \mathrm{~B} ; \mathrm{P}=0.0073$ ) and $\mathrm{BV} / \mathrm{TV}$ ratio
(Fig. 3C; $\mathrm{P}=0.0023$ ) in the tissues of the PTKO model group were significantly lower when compared with those of the control group. As shown in Fig. 3D, trabecular bone spacing in the PTKO model group was higher in comparison with that of the control group $(\mathrm{P}=0.0042)$. Low myrtol treatment did not markedly affect these four parameters in PTKO mice (Fig. 3). However, treatment with medium and high dose myrtol significantly reversed the subchondral bone thickness $(\mathrm{P}=0.0077$ and $\mathrm{P}=0.0062$, respectively), subchondral bone density $(\mathrm{P}=0.0095$ and $\mathrm{P}=0.0089$, respectively), $\mathrm{BV} / \mathrm{TV}(\mathrm{P}=0.0081$ and $\mathrm{P}=0.0058$, 
A

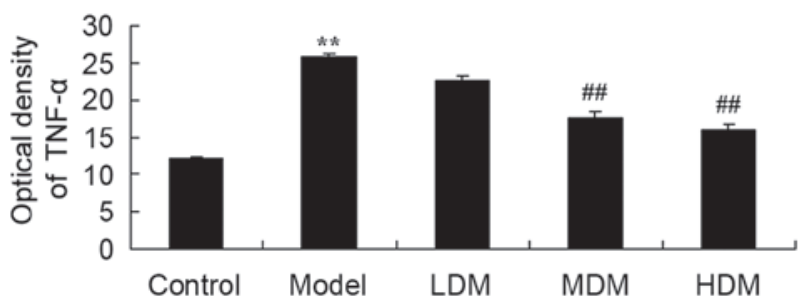

B

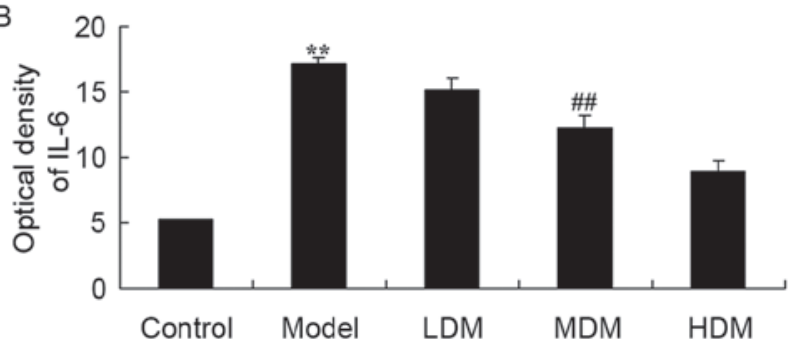

Figure 4. Myrtol improves the activities of (A) TNF- $\alpha$ and (B) IL-6 in a PTKO mouse model, as observed by ELISA. ${ }^{* *} \mathrm{P}<0.01$ vs. control model group; $\#$ P $<<0.01$ vs. PTKO group. PTKO, post-traumatic knee osteoarthritis; LDM, low dose myrtol; MDM, medium dose myrtol; HDM, high dose myrtol; TNF, tumor necrosis factor; IL, interleukin.

A

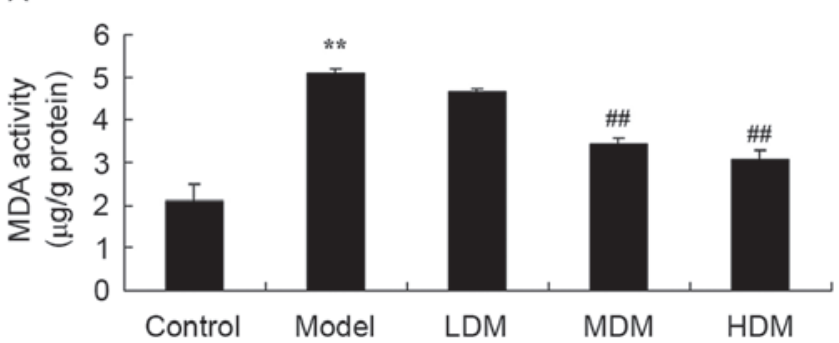

B

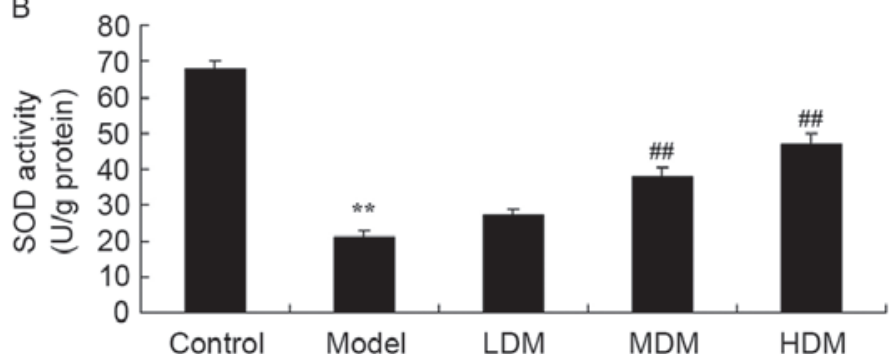

Figure 5. Myrtol improves the levels of (A) MDA and (B) SOD in PTKO model, as observed by ELISA. ${ }^{* *} \mathrm{P}<0.01$ vs. control model group; ${ }^{\# \#} \mathrm{P}<0.01 \mathrm{vs}$. PTKO group. PTKO, post-traumatic knee osteoarthritis; LDM, low dose myrtol; MDM, medium dose myrtol; HDM, high dose myrtol; MDA, malondialdehyde; SOD, superoxide dismutase.

respectively) and trabecular bone spacing $(\mathrm{P}=0.0047$ and $\mathrm{P}=0.0031$, respectively.) in PTKO mice (Fig. 3A-D).

Myrtol improves the activities of TNF- $\alpha$ and IL- 6 in the PTKO model. To explore the effect of myrtol on the levels of inflammation factors in the PTKO model, the activities of TNF- $\alpha$ and IL- 6 were measured using ELISA kits. As shown in Fig. 4, the activities of TNF- $\alpha(\mathrm{P}=0.0025)$ and IL-6 $(\mathrm{P}=0.0013)$ in the PTKO model group were significantly increased when compared with those of the control group. Treatment with medium or high dose myrtol significantly reduced the TNF- $\alpha$ (Fig. 4A; $\mathrm{P}=0.0054$ and $\mathrm{P}=0.0048$ ) and IL-6 (Fig. 4B; $\mathrm{P}=0.0066$ and $\mathrm{P}=0.0032$ ) activities in the $\mathrm{PTKO}$ mice. By contrast, TNF- $\alpha$ and IL- 6 activities in the low dose myrtol treatment group were not evidently altered compared with the model group (Fig. 4).

Myrtol improves the levels of MDA and SOD in the PTKO model. To further explore the effect of myrtol on oxidative stress in the PTKO mouse model, the activities of MDA and SOD were measured using ELISA kits. As shown in Fig. 5, the activity of MDA $(\mathrm{P}=0.0031)$ was increased and the activity of SOD $(\mathrm{P}=0.0027)$ was reduced in the PTKO model group in comparison with those of the control group. Treatment with medium or high dose myrtol significantly reversed the PTKO-altered MDA ( $\mathrm{P}=0.0068$ and $\mathrm{P}=0.0055$, respectively) and $\operatorname{SOD}(\mathrm{P}=0.0042$ and $\mathrm{P}=0.0031$, respectively) activities, while low dose treatment had no significant effect in PTKO mice (Fig. 5).

Myrtol improves the level of TGF- $\beta 1$ in the PTKO model. To examine the mechanism underlying the action of myrtol

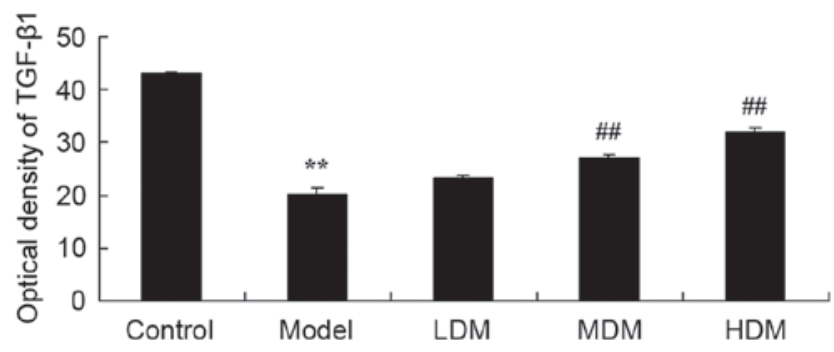

Figure 6. Myrtol improves the level of TGF- $\beta 1$ in a PTKO mouse model, as determined using ELISA. ${ }^{* *} \mathrm{P}<0.01$ vs. control model group; ${ }^{\# \#} \mathrm{P}<0.01$ vs. PTKO group. PTKO, post-traumatic knee osteoarthritis; LDM, low dose myrtol; MDM, medium dose myrtol; HDM, high dose myrtol; TGF, transforming growth factor.

treatment in PTKO, TGF- $\beta 1$ level was detected using an ELISA kit. As shown in Fig. 6, there was a significant increase in TGF- $\beta 1$ level of the PTKO model group, compared with the control group $(\mathrm{P}=0.0039)$. In the medium and high myrtol treatment groups, the TGF- $\beta 1$ activation was significantly inhibited compared with the PTKO model group (Fig. 6; $\mathrm{P}=0.0066$ and $\mathrm{P}=0.0021$, respectively).

Myrtol improves the level of ROS in the PTKO model. The effect of myrtol on PTKO-induced ROS generation was determined using an ELISA kit, in order to analyze the underlying mechanism of myrtol on PTKO. As shown in Fig. 7, the ROS level in the PTKO model group was markedly increased compared with that of the control group $(\mathrm{P}=0.0018)$. Treatment with medium or high dose myrtol significantly reduced the PTKO-induced ROS level in PTKO mice (Fig. 7; P=0.0083 and $\mathrm{P}=0.0041$, respectively). 


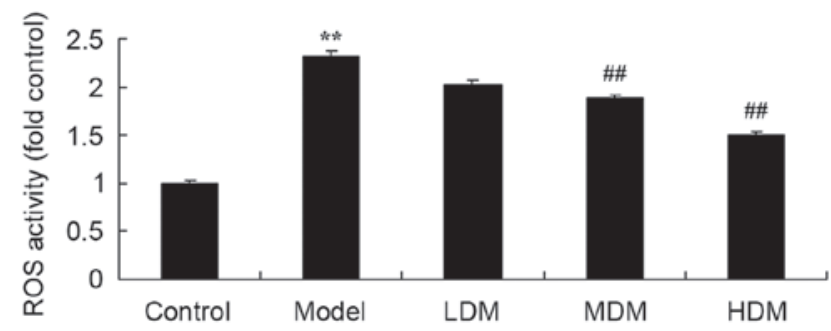

Figure 7. Myrtol improves the level of ROS in a PTKO mouse model, as determined using ELISA. ${ }^{* *} \mathrm{P}<0.01$ vs. control model group; ${ }^{\# \#} \mathrm{P}<0.01$ vs. PTKO group. PTKO, post-traumatic knee osteoarthritis; LDM, low dose myrtol; MDM, medium dose myrtol; HDM, high dose myrtol; ROS, reactive oxygen species.

A

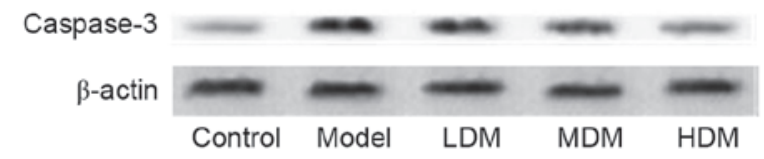

B

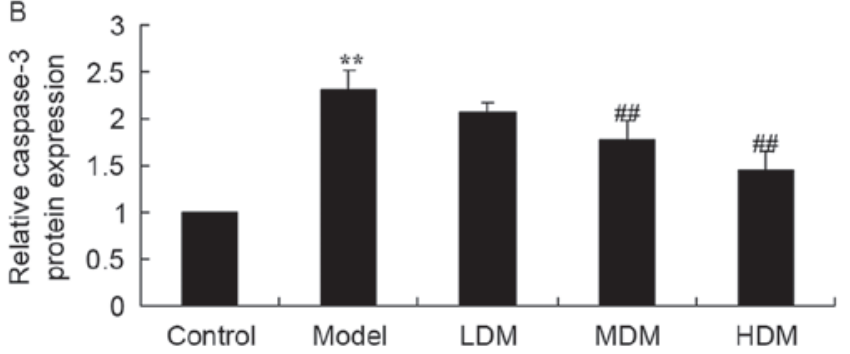

Figure 8. Myrtol improves the level of caspase-3 protein in the PTKO model, as observed in the (A) western blots and (B) the quantified caspase- 3 protein expression. ${ }^{* *} \mathrm{P}<0.01$ vs. control model group; ${ }^{\# \#} \mathrm{P}<0.01$ vs. $\mathrm{PTKO}$ group. PTKO, post-traumatic knee osteoarthritis; LDM, low dose myrtol; MDM, medium dose myrtol; HDM, high dose myrtol.

Myrtol improves the level of caspase-3 protein in the PTKO model. To examine the mechanism of myrtol on the apoptosis of PTKO, caspase-3 protein was measured using western blot analysis. As shown in Fig. 8, the level of caspase-3 protein in PTKO model mice was higher than that of the control group $(\mathrm{P}=0.0025)$. Medium or high dose myrtol significantly suppressed the $\mathrm{PTKO}$-induced caspase-3 protein in $\mathrm{PTKO}$ mice (Fig. 8; $\mathrm{P}=0.0076$ and $\mathrm{P}=0.0046$, respectively).

Myrtol improves the level of Bax protein in the PTKO model. To further examine the mechanism of myrtol on apoptosis in PTKO mice, Bax protein expression was measured using western blot analysis. As shown in Fig. 9, the level of Bax protein in the PTKO model was significantly increased compared with that of the control group $(\mathrm{P}=0.0031)$. Medium or high dose myrtol treatment significantly suppressed the PTKO-induced Bax protein in the PTKO mice (Fig. 9; $\mathrm{P}=0.0082$ and $\mathrm{P}=0.0059$, respectively).

\section{Discussion}

PTKO is a frequently-occurring disease that is characterized of soft tissue injury around the joint region, which can lead to articular cartilage injury and eventually involve the entire joint, resulting in articular cartilage degeneration, fibrosis and
A
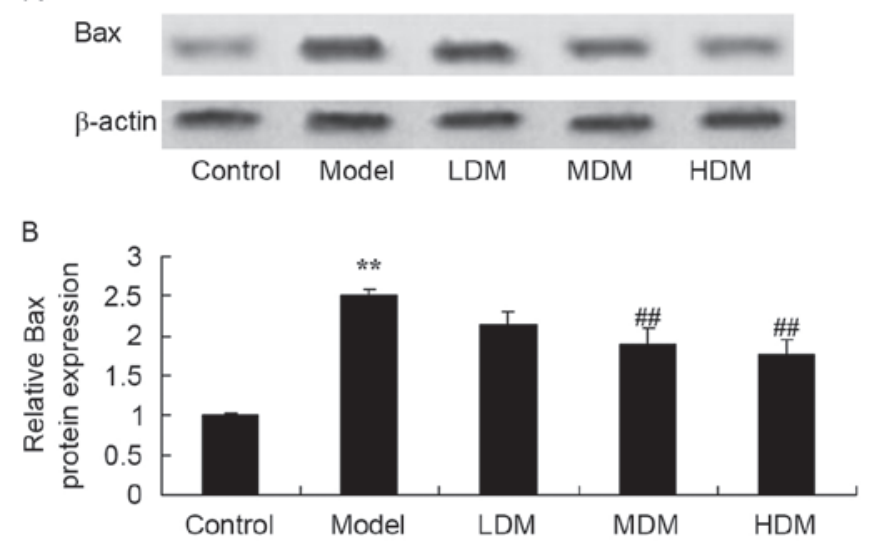

Figure 9. Myrtol improves the level of Bax protein in PTKO model, as observed in the (A) western blots and (B) the quantified Bax protein expression. ${ }^{* *} \mathrm{P}<0.01$ vs. control model group; ${ }^{\# /} \mathrm{P}<0.01$ vs. $\mathrm{PTKO}$ group. $\mathrm{PTKO}$, post-traumatic knee osteoarthritis; LDM, low dose myrtol; MDM, medium dose myrtol; HDM, high dose myrtol.

fracture, and anabrosis (16). Knee pain, stiffness and muscular movement dysfunction are the main clinical manifestations of PTKO (17). Among them, myodynamia reduction of quadriceps femoris muscles is a characteristic manifestation of PTKO muscle dysfunction (18). In the present study, myrtol treatment significantly improved knee osteoarthritis, inhibited the osteoarthritis score, and reversed the PTKO-induced changes in subchondral bone thickness, subchondral bone density, BV/TV and trabecular bone spacing. These findings are in agreement with a previous study demonstrating that myrtol ameliorated cartilage lesions by downregulating the expression of TNF- $\alpha$, IL-6 and Bax in an osteoarthritis rat model (19).

Activation of IL-1 $\beta$ induces damage and synovial inflammation and is an important cytokine causing articular cartilage damage (20). Activation of IL-1 $\beta$ promotes proliferation and differentiation of synovial cells, and disintegration of the cartilage matrix and local immune complex. Free collagen and other decomposition products stimulate the synthesis of IL-1 $\beta$, which forms a vicious circle (20). Furthermore, TNF- $\alpha$ can synthesize PGE2 and collagenase in decomposition lesions, lead to the absorption and destruction of the bone and cartilage (11), as well as promote hyperplasia of fibroblasts. Although IL-6 cannot stimulate synovial cells, chondrocytes synthesize PGE2 and collagenase. However, IL- $1 \beta$ and TNF- $\alpha$ can induce the production of IL-6. Consequently, IL-6, as an inflammatory factor, is considered to be an amplification factor of IL-1 $\beta$ and TNF- $\alpha$. In the current study, myrtol treatment significantly reduced the TNF- $\alpha$ and IL- 6 activities in PTKO mice. Similarly, Beuscher et al (12) revealed that myrtol standardized inhibited inflammatory (TNF- $\alpha$ and IL-6) and allergic mediators.

In the cartilage of PTKO mice, expression quantity has been upregulated to a certain degree, which may initiate the self-rehabilitation of cartilage successively after the cartilage damage. Thus, upregulation of TGF- $\beta 1$ gene and protein promotes downstream signal transduction and regulates the proliferation and differentiation of cartilage (6). Under the effects of specific inflammatory mediators and tumor necrosis factors, the self-rehabilitation ability of cartilage has been 
restricted, so that the cartilage cannot be fully repaired (6). In the present study, myrtol treatment significantly inhibited the activation of TGF- $\beta 1$ level in PTKO mice. Zhao et al (13) also observed that myrtol standardized inhibited TGF- $\beta 1$ expression, and suppressed the levels of TNF- $\alpha$, IL-1 $\beta$ and IL- 6 in radiation-induced lung injury.

The damage of PTKO articular cartilage and bone tissue are closely associated with abnormalities of the cell apoptosis process. Over-proliferation of synovial cells is originated from comparative deficiency of synovial cell apoptosis (21). In recent years, research has detected that the second messenger ROS is associated with PTKO damage (22). The oxidative stress caused by ROS is able to damage mitochondrial respiratory chain. Damaged mitochondria can lead to cell senescence and death. However, ROS generation has an important effect on the over-proliferation of PTKO and synovial membrane (23). Previous studies have suggested that ROS can regulate cell proliferation, differentiation, programmed cell death and aging. A small increase in ROS level promotes cell proliferation $(22,24)$, while a moderate increase can induce cell apoptosis, and high ROS levels directly cause necrocytosis. High concentration of ROS can directly or indirectly damage the mitochondrial membrane structure, induce voltage reduction of mitochondrial membrane, and lead to mitochondrial bulging (21). Mitochondrial bulging indicates that the changes of mitochondrial membrane structure will result in loss of the ATP synthesis ability (24). In the present study, myrtol treatment significantly reduced the PTKO-induced ROS level, and reversed the PTKO-altered MDA and SOD activities in a mouse model. Similarly, Rantzsch et al (25) reported that myrtol had effective antioxidative properties in patients with chronic obstructive pulmonary disease.

Cell apoptosis occurs in various physiological and pathological processes, and is a cell death program with strict regulation of energy dependence (26). In particular, Bax is the 'molecular switch' initiating cell apoptosis, and serves a key function on determines cell apoptosis (27). In addition, caspase is not only an important molecule in apoptosis, but also serves as a key protease of Bax (28). Activation of the caspase decomposition specificity substrate may lead to cell apoptosis (29). The present study found that myrtol significantly suppressed PTKO-induced caspase-3 and Bax protein expression in PTKO mice. A study by Ying et al (19) also demonstrated that myrtol ameliorates cartilage lesions by downregulating the expression of Bax in an osteoarthritis rat model.

In conclusion, myrtol treatment improved knee osteoarthritis, inhibited the osteoarthritis score, and reversed the PTKO-induced changes in subchondral bone thickness, subchondral bone density, BV/TV and trabecular bone spacing in a mouse model of PTKO. Myrtol also inhibited inflammation and oxidative stress, reduced ROS and TGF- $\beta 1$ levels, and suppressed caspase- 3 and Bax protein expression in PTKO mice. These results indicated that myrtol improves PTKO and may be a promising drug for clinical therapy.

\section{References}

1. Swärd P, Fridén T, Boegård T, Kostogiannis I, Neuman P and Roos H: Association between varus alignment and post-traumatic osteoarthritis after anterior cruciate ligament injury. Knee Surg Sports Traumatol Arthrosc 21: 2040-2047, 2013
2. Wolsko PM, Eisenberg DM, Simon LS, Davis RB, Walleczek J, Mayo-Smith M, Kaptchuk TJ and Phillips RS: Double-blind placebo-controlled trial of static magnets for the treatment of osteoarthritis of the knee: Results of a pilot study. Altern Ther Health Med 10: 36-43, 2004.

3. Giannini S, Buda R, Ruffilli A, Pagliazzi G, Ensini A, Grigolo B, Desando G and Vannini F: Failures in bipolar fresh osteochondral allograft for the treatment of end-stage knee osteoarthritis. Knee Surg Sports Traumatol Arthrosc 23: 2081-2089, 2015.

4. Jiang Q, Qiu YT, Chen MJ, Zhang ZY and Yang C: Synovial TGF- $\beta 1$ and MMP-3 levels and their correlation with the progression of temporomandibular joint osteoarthritis combined with disc displacement: A preliminary study. Biomed Rep 1: 218-222, 2013.

5. Wilbers RH, Westerhof LB, van Raaij DR, van Adrichem M, Prakasa AD, Lozano-Torres JL, Bakker J, Smant G and Schots A: Co-expression of the protease furin in Nicotiana benthamiana leads to efficient processing of latent transforming growth factor- $\beta 1$ into a biologically active protein. Plant Biotechnol J 14: 1695-1704, 2016.

6. Fahlgren A, Andersson B and Messner K: TGF-beta1 as a prognostic factor in the process of early osteoarthrosis in the rabbit knee. Osteoarthritis Cartilage 9: 195-202, 2001.

7. Vonwil D, Trüssel A, Haupt O, Gobaa S, Barbero A, Shastri VP and Martin I: Substrate elasticity modulates TGF beta stimulated re-differentiation of expanded human articular chondrocytes. Drug Deliv Transl Res 2: 351-362, 2012.

8. Heard BJ, Solbak NM, Achari Y, Chung M, Hart DA, Shrive NG and Frank CB: Changes of early post-traumatic osteoarthritis in an ovine model of simulated ACL reconstruction are associated with transient acute post-injury synovial inflammation and tissue catabolism. Osteoarthritis Cartilage 21: 1942-1949, 2013.

9. Lewis JS, Hembree WC, Furman BD, Tippets L, Cattel D, Huebner JL, Little D, DeFrate LE, Kraus VB, Guilak F and Olson SA: Acute joint pathology and synovial inflammation is associated with increased intra-articular fracture severity in the mouse knee. Osteoarthritis Cartilage 19: 864-873, 2011.

10. Bradley EW, Carpio LR, McGee-Lawrence ME, Castillejo Becerra C, Amanatullah DF, Ta LE, Otero M, Goldring MB, Kakar S and Westendorf JJ: Phlppl facilitates post-traumatic osteoarthritis and is induced by inflammation and promoter demethylation in human osteoarthritis. Osteoarthritis Cartilage 24: 1021-1028, 2016.

11. Huebner KD, Shrive NG and Frank CB: Dexamethasone inhibits inflammation and cartilage damage in a new model of post-traumatic osteoarthritis. J Orthop Res 32: 566-572, 2014.

12. Beuscher N, Kietzmann M, Bien E and Champeroux P: Interference of myrtol standardized with inflammatory and allergic mediators. Arzneimittelforschung 48: 985-989, 1998.

13. Zhao DY, Qu HJ, Guo JM, Zhao HN, Yang YY, Zhang P, Cao K, Lei X, Cui JG, Liu C, et al: Protective effects of Myrtol standardized against radiation-induced lung injury. Cell Physiol Biochem 38: 619-634, 2016.

14. Christiansen BA, Anderson MJ, Lee CA, Williams JC, Yik JH and Haudenschild DR: Musculoskeletal changes following non-invasive knee injury using a novel mouse model of post-traumatic osteoarthritis. Osteoarthritis Cartilage 20: 773-782, 2012.

15. Furman BD, Strand J, Hembree WC, Ward BD, Guilak F and Olson SA: Joint degeneration following closed intraarticular fracture in the mouse knee: A model of posttraumatic arthritis. J Orthop Res 25: 578-592, 2007.

16. Bégué T, Mebtouche N and Levante S: One-stage procedure for total knee arthroplasty in post-traumatic osteoarthritis of the knee with wound defect. Usefulness of navigation and flap surgery. Knee 19: 948-950, 2012.

17. Nordenvall R, Bahmanyar S, Adami J, Mattila VM and FelländerTsai L: Cruciate ligament reconstruction and risk of knee osteoarthritis: The association between cruciate ligament injury and post-traumatic osteoarthritis. a population based nationwide study in Sweden, 1987-2009. PLoS One 9: e104681, 2014.

18. Bala A, Penrose CT, Seyler TM, Mather RC 3rd, Wellman SS and Bolognesi MP: Outcomes after total knee arthroplasty for post-traumatic arthritis. Knee 22: 630-639, 2015.

19. Ying B, Maimaiti AK, Song D and Zhu S: Myrtol ameliorates cartilage lesions in an osteoarthritis rat model. Int J Clin Exp Pathol 8: 1435-1442, 2015.

20. Satkunananthan PB, Anderson MJ, De Jesus NM, Haudenschild DR, Ripplinger CM and Christiansen BA: In vivo fluorescence reflectance imaging of protease activity in a mouse model of post-traumatic osteoarthritis. Osteoarthritis Cartilage 22: 1461-1469, 2014. 
21. Jiang L, Li L, Geng C, Gong D, Jiang L, Ishikawa N, Kajima K and Zhong L: Monosodium iodoacetate induces apoptosis via the mitochondrial pathway involving ROS production and caspase activation in rat chondrocytes in vitro. J Orthop Res 31: 364-369, 2013.

22. Courties A, Gualillo O, Berenbaum F and Sellam J: Metabolic stress-induced joint inflammation and osteoarthritis. Osteoarthritis Cartilage 23: 1955-1965, 2015.

23. Ziskoven C, Jäger M, Zilkens C, Bloch W, Brixius $\mathrm{K}$ and Krauspe R: Oxidative stress in secondary osteoarthritis: From cartilage destruction to clinical presentation? Orthop Rev (Pavia) 2: e23, 2010.

24. Xie L, Lin AS, Kundu K, Levenston ME, Murthy N and Guldberg RE: Quantitative imaging of cartilage and bone morphology, reactive oxygen species, and vascularization in a rodent model of osteoarthritis. Arthritis Rheum 64: 1899-1908, 2012.

25. Rantzsch U, Vacca G, Dück R and Gillissen A: Anti-inflammatory effects of Myrtol standardized and other essential oils on alveolar macrophages from patients with chronic obstructive pulmonary disease. Eur J Med Res 14 (Suppl 4): S205-S209, 2009.
26. Bar-Yehuda S, Rath-Wolfson L, Del Valle L, Ochaion A, Cohen S, Patoka R, Zozulya G, Barer F, Atar E, Piña-Oviedo S, et al: Induction of an antiinflammatory effect and prevention of cartilage damage in rat knee osteoarthritis by CF101 treatment. Arthritis Rheum 60: 3061-3071, 2009.

27. Li H, Lei M, Yu C, Lv Y, Song Y and Yang L: Mechano growth factor-E regulates apoptosis and inflammatory responses in fibroblast-like synoviocytes of knee osteoarthritis. Int Orthop 39: 2503-2509, 2015.

28. Lee CH, Wen ZH, Chang YC, Huang SY, Tang CC, Chen WF, Hsieh SP, Hsieh CS and Jean YH: Intra-articular magnesium sulfate $(\mathrm{MgSO} 4)$ reduces experimental osteoarthritis and nociception: Association with attenuation of N-methyl-D-aspartate (NMDA) receptor subunit 1 phosphorylation and apoptosis in rat chondrocytes. Osteoarthritis Cartilage 17: 1485-1493, 2009.

29. Bouderlique T, Vuppalapati KK, Newton PT, Li L, Barenius B and Chagin AS: Targeted deletion of Atg 5 in chondrocytes promotes age-related osteoarthritis. Ann -Rheum Dis 75: 627-631, 2016. 